\title{
INVESTIGATION OF A NEW MODEL FOR BUBBLY CAVITATING FLOW
}

\author{
Tim Colonius \\ Division of Engineering and Applied Science, \\ California Institute of Technology, \\ Pasadena, California 91125, USA
}

\author{
Daniel Fuster \\ CNRS (UMR 7190), Université Pierre et Marie Curie. \\ Institut Jean le Rond d'Alembert, France
}

\section{SUMMARY}

A new model for bubbly, cavitating flow is validated and used to study the shock-induced oscillations of bubble clouds arising in shockwave lithotripsy and other applications. Compared to previous models based on volume and phase averaging, the new model extends the range of void fractions that can be reliably simulated and, for appropriately low void fractions, reproduces the results of the polydisperse phase-averaged model with much smaller computational expense.

\section{INTRODUCTION}

Our overall objective is to understand and predict complex, multiphase, cavitating flow phenomena. Applications include cavitation reloading of surfaces exposed to underwater explosions, shockwave lithotripsy, and high-intensity focused ultrasounds. Bubble clouds in many applications are polydisperse and may involve many thousands of bubbles; it is not computationally tractable to directly resolve both phases. Approximate systems of equations based on a volume or phase-averaged continuum approach are required for these situations. In the most sophisticated of these models, two-way coupled (dynamic) effects of cavitation are considered meaning that the time evolution of representative, spherical bubbles are determined by solving a Rayleigh-Plessettype (RP) equation whose driving pressure is determined from the local pressure of the continuous phase, and whose result effects the continuous phase by altering the void fraction $[1,2]$. Various extensions of the RP model adequately account for phase change, liquid compressibility, and heat and mass transfer within the bubble contents [3], but existing phase- and volume-averaging approaches require two fundamental assumptions that limit their applicability: low void fraction, and scale separation between the typical bubble size and the length scales associated with the (averaged) flow field.

\section{Model}

In this paper, we consider a new bubbly flow methodology based on volume-averaged equations that relaxes the scaleseparation assumption and directly accounts for bubble-bubble interactions. The full equations for the model are detailed in ref [4]; we summarize the key steps in the derivation here. The model utilizes a volume-averaging approach [1]. We solve for the averaged continuity, momentum and energy equations in addition to the advection equation for every component present in the system. These equations can be written as [5]

$$
\begin{array}{r}
\frac{\partial \alpha_{i} \rho_{i}}{\partial t}+\nabla \cdot\left(\alpha_{i} \rho_{i} u\right)=0 \quad i=1, \ldots, N, \\
\frac{\partial \rho u}{\partial t}+\nabla \cdot(\rho u u+p I)=0, \\
\frac{\partial \rho e}{\partial t}+\nabla \cdot(u(\rho e+p))=0, \\
\frac{\partial \alpha_{i}}{\partial t}+u \cdot \nabla \alpha_{i}=0 \quad i=1, \ldots, N-1,
\end{array}
$$

where

$$
\sum_{i=1}^{N} \alpha_{i}=1
$$

This set of equations is closed by specifying an equation of state. In this case we assume that this equation obeys the following form

$$
p \Gamma+\Pi_{\infty}=\rho e-\frac{1}{2} \rho\|u\|^{2} .
$$

This model differs from previous models in how the void fraction, $\beta$, is defined in terms of the bubble radius, and how the average pressure in a computational cell relates to the pressure felt by the corresponding bubbles in that cell. Bubbles are treated as point particles that are either advected with the flow, or, in cases where relative motion is important, with their own equations of motion with forces determined by empirical drag relations. If a polydisperse mixture is desired, then we set the initial radii of each bubble according to a representative probability density function. To compute $\beta$, we multiply each individual bubble volume by discrete delta function that smears its value onto the computational grid, and, at each point in the domain, sum 


\section{Proceedings of the Eighth International Symposium on Cavitation (CAV 2012)}

this over all bubbles. To treat the dynamics of each bubble, we develop a new RP-like equation that accounts for a cluster of neighboring bubbles in the vicinity of each bubble. This equation is a generalization of the incompressible bubble-cluster model of Ilinskii et al. [6] to compressible liquids, and likewise assumes spherical bubbles and potential flow in the vicinity of the bubbles. The key step in the derivation is to represent the pressure far from the cluster (the pressure at infinity, as seen by the bubbles) in the potential flow model in terms of the average pressure of the liquid within a computational cell. From the potential flow solution for the bubble cluster, we may expand the pressure at infinity in terms of the cell pressure plus a correction term that can be estimated based on the bubble dynamics. The correction term thus represents a modification to the local value of pressure associated with the bubble dynamics. If the void fraction is sufficiently small, this term also becomes small and the equations relax to the previous volume-averaged equations. By taking a cell based approach rather than a multiple-scales approach, the model we obtain is grid dependent, much in the same way a Large Eddy Simulation of turbulence depends on the grid spacing. In the limit of the grid spacing going to zero, the LES model switches off, resulting in a direct numerical simulation of the turbulent flow. In the present model, as the grid is refined to the size of an individual bubble, the model exactly represents the dynamics of an isolated spherical bubble. This is verified in detail by comparing against theoretical solutions for this case [4]. At the other extreme, the model is demonstrated to converge to the solution to ensemble-averaged models for low spatial resolution. However, the computational expense is greatly reduced compared to the corresponding ensemble-averaged approach. Therefore, the current model seems suitable to numerically investigate complex problems where the computational effort of current ensemble-averaged models is prohibitive.

At intermediate resolutions, the model provides information about the instantaneous local pressures induced by bubble dynamics that is missing from ensemble-averaged approaches. Thus it also seems advantageous for considering cavitation damage and fluid structure interaction problems where structures respond to the instantaneous pressures reached near bubble rather than averaged ones.

\section{Numerical Method}

The new cavitation model presented in ref [4] has been incorporated into a state-of-the-art multi-fluid, compressible flow solver that utilizes advanced WENO shock-capturing techniques [5]. This code permit us to simulate violent cloud collapse with its associated shock waves. Because the gas/vapor phase can be considered as disperse and the density of this phase is much smaller than that of the continuous phase, we can solve for the averaged energy, momentum, continuity equations assuming that

$$
\begin{array}{r}
\rho \approx \rho_{b}(1-\beta), \\
\rho u \approx(1-\beta) \rho_{b} u, \\
\rho e \approx(1-\beta) \rho_{b} e_{b},
\end{array}
$$

where the subscript $b$ is used to denote the "bulk phase". In a system with $N_{b}$ "bulk phases", the volume fraction must satisfy that

$$
\sum_{i=1}^{N_{b}} \alpha_{i}+\beta=1
$$

We define a corrected concentration of the $i$ th component as

$$
\alpha_{i}^{\prime}=\frac{\alpha_{i}}{(1-\beta)}
$$

such that we satisfy the condition

$$
\sum_{i=1}^{N_{b}} \alpha_{i}^{\prime}=1
$$

It can be verified, that because the advection equation is satisfied for $\beta$, we can write

$$
\frac{\partial \alpha_{i}^{\prime}}{\partial t}+u \cdot \nabla \alpha_{i}^{\prime}=0 \quad i=1, \ldots, N_{b}-1
$$

Then, we can solve for the $N_{b}$ equations of mass (Eqs. 1) defining a new density for the $i$ th compound as

$$
\rho_{i}^{\prime}=\rho_{i}(1-\beta)
$$

such that $\rho_{i} \alpha_{i}=\rho_{i}^{\prime} \alpha_{i}^{\prime}$. Thus, we write

$$
\frac{\partial \alpha_{i}^{\prime} \rho_{i}^{\prime}}{\partial t}+\nabla \cdot\left(\alpha_{i}^{\prime} \rho_{i}^{\prime} u\right)=0 \quad i=1, \ldots, N_{b}
$$

We note that the averaged density is recovered

$$
\rho=\sum_{i=1}^{N_{b}} \alpha_{i}^{\prime} \rho_{i}^{\prime} .
$$

Using Eq. 11, we rewrite Eq. 15 as

$$
\frac{\partial \rho_{i} \alpha_{i}^{\prime}}{\partial t}+\nabla \cdot\left(\rho_{i} \alpha_{i}^{\prime} u\right)=\frac{\rho_{i} \alpha_{i}^{\prime}}{1-\beta}\left[\frac{\partial \beta}{\partial t}+u \cdot \nabla \beta\right] \quad i=1, \ldots, N_{b}
$$

This formulation is convenient because the terms related to the bubble motion and translation are grouped in the right hand side of the equation. The temporal derivative of $\beta$ is

$$
\frac{\partial \beta}{\partial t}=\sum_{i}\left[\frac{\partial V_{i}}{\partial t} \Upsilon(d)+V_{i} \frac{\partial \Upsilon(d)}{\partial t}\right]
$$


where $V_{i}=4 / 3 \pi R_{i}^{3}$. As we only allow spherical oscillations, this expression can be further simplified to

$$
\frac{\partial V_{i}}{\partial t}=4 \pi R_{i}^{2} \dot{R}_{i}
$$

Finally, applying the chain rule, the temporal derivative of the symmetric kernel can be expressed as

$$
\frac{\partial \Upsilon(d)}{\partial t}=-u_{p} \cdot \nabla \Upsilon(d)
$$

where $u_{p}$ denotes the bubble velocity represented as a Lagrangian particle. Thus equation 17 can be rewritten as

$$
\frac{\partial \rho_{i} \alpha_{i}^{\prime}}{\partial t}+\nabla \cdot\left(\rho_{i} \alpha_{i}^{\prime} u\right)=\frac{\rho_{i} \alpha_{i}^{\prime}}{1-\beta}\left[\frac{\partial \beta}{\partial t}+u \cdot \nabla \beta\right] \quad i=1, \ldots, N_{b}
$$

Following a similar approach, we decide to express the averaged equations as a function of the "bulk" averaged properties $\left(\rho_{b}\right.$ and $e_{b}$ ) instead of the averaged properties ( $\rho$ and $e$ ). Thus, the averaged momentum equation reads

$$
\frac{\partial \rho_{b} u}{\partial t}+\nabla \cdot\left(\rho_{b} u u\right)+\frac{1}{1-\beta} \nabla \cdot(p I)=\frac{\rho_{b} u}{1-\beta}\left[\frac{\partial \beta}{\partial t}+u \cdot \nabla \beta\right] .
$$

and analogously, for the energy equation we find

$$
\frac{\partial \rho_{b} e_{b}}{\partial t}+\nabla \cdot\left(u \rho_{b} e_{b}\right)+\frac{1}{1-\beta} \nabla \cdot(u p)=\frac{\rho_{b} e_{b}}{1-\beta}\left[\frac{\partial \beta}{\partial t}+u \cdot \nabla \beta\right] .
$$

Finally, the pressure is directly obtained using the energy values obtained from the solution of the Eq. 23

$$
p \Gamma+\Pi_{\infty}=\rho_{b} e_{b}-\frac{1}{2} \rho_{b}\|u\|^{2} .
$$

\section{RESULTS}

\section{Linear regime}

As a validation of the model and its numerical implementation, we consider acoustic wave propagation in a polydisperse bubbly fluid, and compare the results to the continuum bubbly flow model of Ando et al [7]. We consider a homogeneous region of bubbles where $\beta=0.1 \%$ and averaged radius $\bar{R}=10 \mu \mathrm{m}$. The nuclei distribution obeys the same log-normal distribution with $\sigma=0.7$ as the one used by Ando et al [7]. We consider a rectangular domain of dimensions $\frac{L_{x}}{\bar{R}} \times \frac{L_{y}}{\bar{R}}=7000 \times 500$, with grid spacing $\Delta x / \bar{R}=2.5, \Delta y / \bar{R}=40$, and $\Delta z / \bar{R}=166$. A onedimensional Gaussian pulse is initialized at $x=0$ as

$$
p(x)=p_{0}\left(1+\varepsilon \exp \left[-x^{2} / \alpha^{2}\right]\right) .
$$

To generate a linear response, we use a small $\varepsilon=10^{-4}$. We also keep the pulse narrow $\left(\frac{\sigma}{\bar{R}}=1\right)$ to excite a range of frequencies. In order to obtain a representative behavior of the averaged bubble propagation, the pressure disturbance is averaged in a plane perpendicular to the wave propagation. Thus, we define the pressure perturbation $p^{\prime}$ at a giving sampling point $x_{i}$ as

$$
p^{\prime}\left(x_{i}\right)=\frac{\iint\left(p\left(x_{i}, y, z\right)-p_{0}\right) d y d z}{\iint d y d z} .
$$

Then, following ref [8], the attenuation and speed of sound are obtained using the Fourier Transform of the temporal evolution of $p^{\prime}\left(x_{i}\right)$ at multiple sampling points. The values for the complex and real part of the complex wavenumber are fitted by least squares using the data from five sampling points equally spaced. In particular, two different sets are used; the first ones are equally spaced with $\Delta s / \bar{R}=5$ in order to correctly capture the speed of sound in the high frequency range, another set of points is chosen with $\Delta s / \bar{R}=20$. In this later case, as we increase the sampling region, we expect the averaged results to better agree with the linear theory predictions. Figure 1 shows a good agreement between the theoretical predictions and the simulation results. As expected, the fitting of the attenuation in the low frequency range improves as we increase the distance between samples.

\section{Non-linear regime}

In this section we present preliminary studies of the growth and collapse of a cloud of cavitation bubbles generated by a lithotripter shock wave. This is an example of the potential of the algorithm to investigate problems involving large numbers of bubbles.

We model a ultrasonic wavepacket encountering an elliptical-shaped cloud of bubbles. The bubble cloud has major axis of $3 \mathrm{~mm}$, a minor axis of $0.5 \mathrm{~mm}$, and it is composed of a log-normal distribution of bubbles with an average radius of 5 $\mu \mathrm{m}$ and a variance of $\sigma=0.7$. The initial void fraction is set to $10^{-4}$. In order to speed up the calculation a $2 \mathrm{D}$ simulation is considered. The wavepacket is generated imposing a volumetric source far from the bubble cloud that radiates periodic waves that are concentrated at the bubble cluster location. The amplitude of the ultrasonic waves at the focal region reach peak pressures of around $150 \mathrm{~atm}$ and negative tensile phases with pressures of the order of $-80 \mathrm{~atm}$.

Figures 2 and 3 represent, at early and later times respectively, snapshots of the void concentration and pressure field obtained with an increasing level of resolution. As the compressive portion of the wave arrives first, bubbles are initially collapsed. Because the void fraction is sufficiently small, the compressive portion of the wave modified little by the bubbles. The tensile part of the wave then results in a sudden bubble expansion that disperses the incident wave and results in scattered waves behind the main wavepacket. Also, the subsequent incident waves are strongly influenced by the dynamics of the bubble cloud due to the significant increase on the void fraction. 

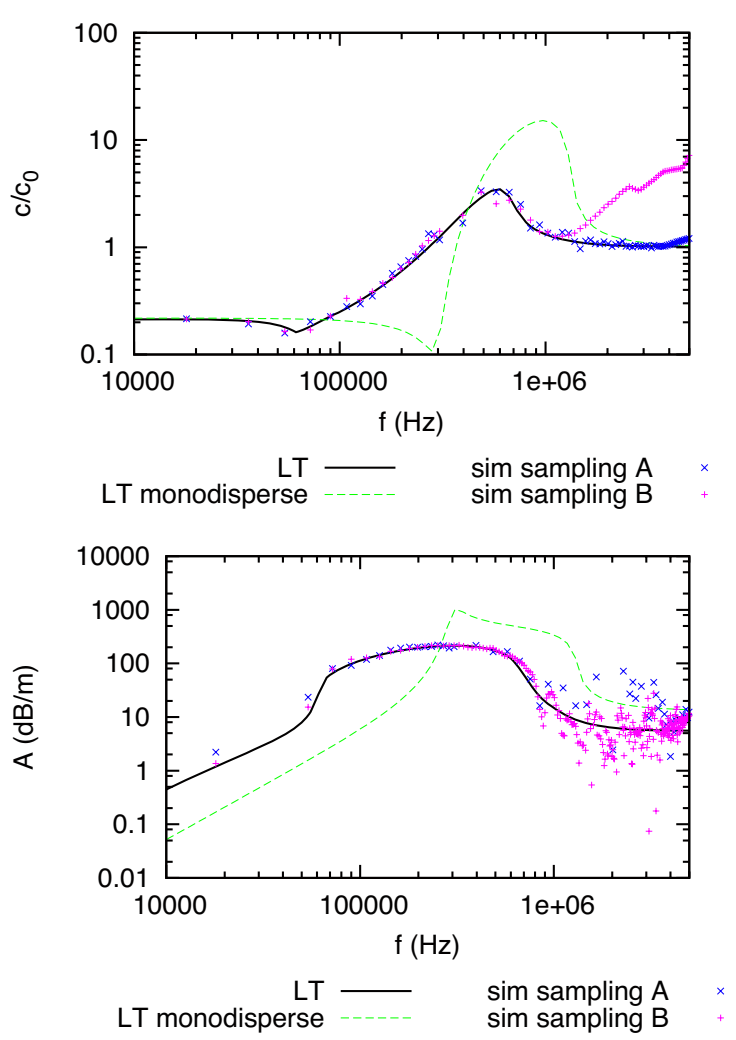

Figure 1. Speed of sound and attenuation of a polydisperse bubble cloud of randomly distributed bubbles obtained from simulation results and comparison with the linear theory predictions of ref [9]. The simulation data is fitted with 5 samples equally spaced with $\Delta s / \bar{R}=5$ (sampling A) and with $\Delta s / \bar{R}=20$ (sampling B).

Comparing the results using different grid resolutions, we observe that the distortion of the large scale structures of the wave are relatively well captured by low resolved simulations. When the mesh resolution is fine enough as to capture the pressure waves induced by individual bubble implosion, we see finerscale scattered waves and shocks in the solution. Thus, the current method seems a promising technique in order to capture the most important mechanisms associated with the bubble collapse with high fidelity, when resolution permits, and in an averaged way at lower resolution.

\section{CONCLUSIONS}

In this work we present new results obtained from the implementation of a cavitation model presented in ref [4] in a multi-fluid, compressible, shock-capturing flow solver. The new implementation has been validated against the linear theory results for low amplitude pressure disturbances propagating through a cluster of polydisperse and randomly distributed bubbles. We also present an example of a bubble cluster excited by a focused wavepacket. For fine enough resolutions the model

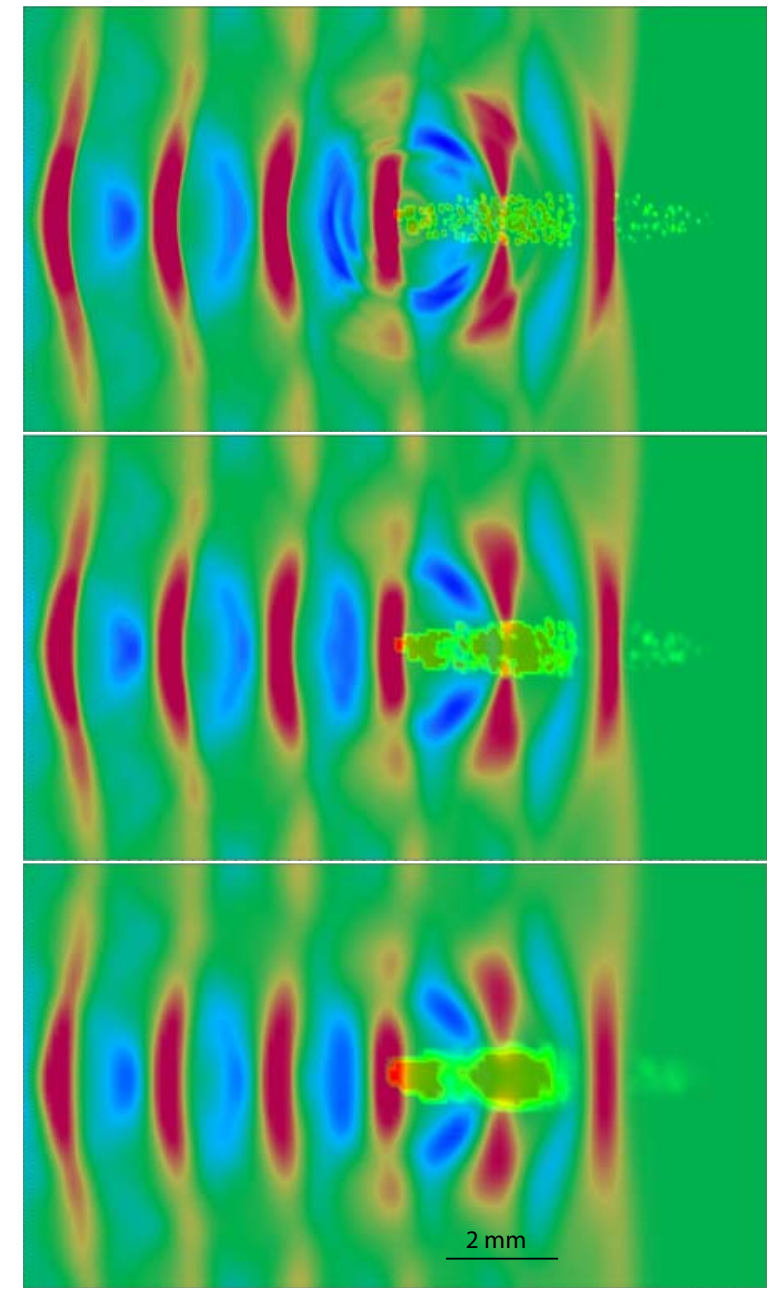

Figure 2. Snapshots of the nondimensional pressure fields $\left(p / p_{0}\right)$ with void fraction superposed at three different mesh resolution: $\Delta x / \bar{R}=$ 6 (top), $\Delta x / \bar{R}=12$ (middle) and $\Delta x / \bar{R}=24$ (bottom).

is able to capture the pressure waves generated during individual bubble collapses. This problem highlights the potential of the technique to obtain predictions of damage or noise associated with bubble collapse. These phenomena are related to high frequency content of the solution that are not properly captured by other averaged models, but which can be obtained, with sufficient resolution, in the present model.

\section{ACKNOWLEDGMENTS}

We gratefully acknowledge the significant contribution from Mr. Vedran Coralic, who provided his multi-component Euler solver for this project. We would like to thank Prof. Keita Ando for providing comparison solutions and, together with Prof Christopher E. Brennen, for their helpful advise. This work 

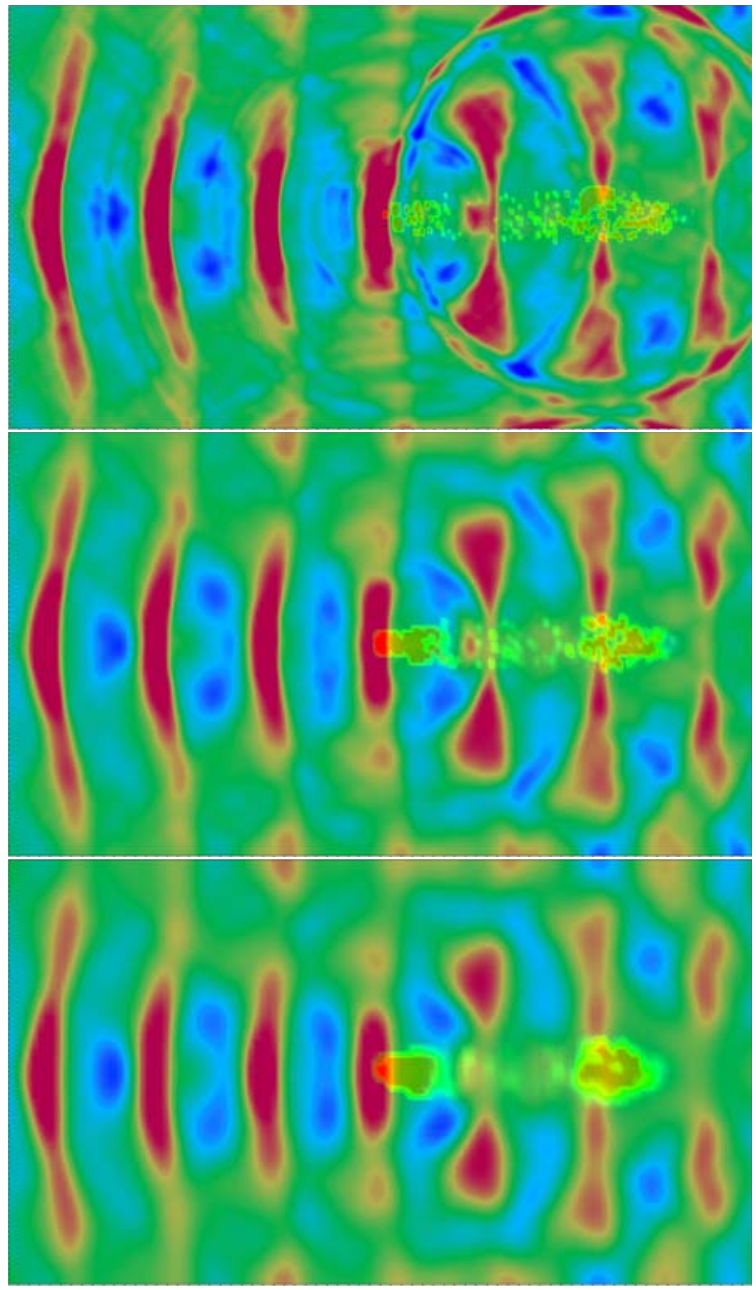

Figure 3. Same as figure 2 but at a later time.

was supported in part by the Spanish Ministry of Education (D. Fuster) and the Office of Naval Research (T. Colonius).

\section{NOMENCLATURE}

\section{Roman symbols}

$e \quad$ internal energy $\left(\mathrm{Jkg}^{-1}\right)$

$N \quad$ Number of species(-)

$p \quad$ pressure $\left(\mathrm{Nm}^{-2}\right)$

$R \quad$ bubble radius (m)

$t \quad$ time (s)

$u \quad$ fluid velocity $\left(\mathrm{ms}^{-1}\right)$

Greek symbols

$\alpha \quad$ species volume fraction(-)

$\beta \quad$ Void fraction of the disperse phase(-)

$\Gamma \quad$ Coefficient for the stiffness equation of state $(-)$.

$\Pi_{\infty} \quad$ 2nd coefficient for the stiffness equation of state $(\mathrm{Pa})$

$\rho \quad$ Density $\left(\mathrm{kgm}^{-3}\right)$ $\sigma \quad$ Polydispersity coefficient $(m)$

Subscripts

b bulk

i ith component

\section{REFERENCES}

[1] Van Wijngaarden, L., 1968. "On the equations of motion for mixtures of liquid and gas bubbles". J. Fluid Mech, 33(3), pp. $465-474$.

[2] Zhang, D., and Prosperetti, A., 1997. "Momentum and energy equations for disperse two-phase flows and their closure for dilute suspensions ". Int. J. Multiphase Flow, 23, pp. 425-453.

[3] Preston, A., Colonius, T., and Brennen, C., 2007. "A reduced order model of diffusive effects on the dynamics of bubbles". Phys. Fluids, 19(123302), pp. 1-19.

[4] Fuster, D., and Colonius, T., 2011. "Modelling bubble clusters in compressible liquids". J. Fluid Mech., 688, pp. 352389.

[5] Johnsen, E., and Colonius, T., 2006. "Implementation of WENO schemes in compressible multicomponent flow problems”. J. Comp. Phys., 219(2), pp. 715-732.

[6] Ilinskii, Y., Hamilton, M., and Zabolotskaya, E., 2007. "Bubble interaction dynamics in Lagrangian and Hamiltonian mechanics". J. Acoust. Soc. Am., 121, p. 786.

[7] Ando, K., Colonius, T., and Brennen, C., 2011. "Numerical simulation of shock propagation in a polydisperse bubbly liquid". Int. J. Mult. Flow, 37, pp. 596-608.

[8] Ando, K., 2010. "Effects of polydispersity in bubbly flows". $\mathrm{PhD}$ Thesis, California Institute of Technology, May 20.

[9] Commander, K., and Prosperetti, A., 1989. "Linear pressure waves in bubbly liquids: Comparison between theory and experiments". J. Acoust. Soc. Am., 85, p. 732. 